\title{
Eye drop technique and patient-reported problems in a real-world population of eye drop users
}

\author{
Els Mehuys ${ }^{1} \cdot$ Christophe Delaey $^{2} \cdot$ Thierry Christiaens $^{3,4} \cdot$ Luc Van Bortel $^{4} \cdot$ Inge Van Tongelen ${ }^{1}$. \\ Jean-Paul Remon ${ }^{1} \cdot$ Koen Boussery ${ }^{1}$
}

Received: 15 January 2019 / Revised: 18 June 2019 / Accepted: 24 September 2019 / Published online: 5 November 2019

(c) The Author(s), under exclusive licence to The Royal College of Ophthalmologists 2019

\begin{abstract}
Objective To assess eye drop technique and patient-reported problems with eye drop instillation in a primary care sample of eye drop users.

Methods Cross-sectional observational study in 136 community pharmacies in Belgium. Patient inclusion criteria were being age $\geq 18$ years and using eye drops for $\geq 1$ month (to ensure that patients were already familiar with eye drop instillation). Participants demonstrated their eye drop technique and completed a self-administered questionnaire.

Results Participants $(n=678)$ had a mean age of $68.9 \pm 12.4$ years. During the demonstration, almost everyone $(98.0 \%)$ successfully instilled at least one drop in the eye, although $14 \%$ required multiple attempts to achieve this. Only $3 \%$ of the sample exhibited perfect drop technique, meaning that they performed correctly all the steps. Most common deviations were touching the bottle to the eye or eyelid (40.7\% of patients), and failing to close the eye $(67.8 \%)$ and perform nasolacrimal occlusion for at least $1 \mathrm{~min}(94.7 \%)$ after drop instillation. Importantly, we found that $20 \%$ of ophthalmic suspensions were not shaken before use. Forty percent of patients reported $\geq 1$ problem with eye drop instillation. Most common problems were difficulties with getting a drop in the eye (18.3\% of patients), too many drops coming out of the bottle (14.6\%), and difficulty squeezing the bottle (12.2\%). About half of the sample recalled having had education in eye drop instillation technique.
\end{abstract}

Conclusion This study showed suboptimal eye drop technique in real-world clinical practice. A proactive role of community pharmacists in detecting and resolving these problems could be helpful.

\section{Introduction}

Topical eye medication is the mainstay of treatment for eye conditions as they are administered directly on the site of action. For patients to gain maximum therapeutic effect

Supplementary information The online version of this article (https:// doi.org/10.1038/s41433-019-0665-y) contains supplementary material, which is available to authorized users.

Els Mehuys

els.mehuys@ugent.be

1 Pharmaceutical Care Unit, Ghent University, Ghent, Belgium

2 Department of Ophthalmology, Maria Middelares Hospital, Ghent, Belgium

3 Department of Public Health and Primary Care, Ghent University, Ghent, Belgium

4 Department of Pharmacology, Ghent University, Ghent, Belgium while minimizing side effects, it is imperative that eye drops are instilled correctly. The most important steps in proper drop technique include instillation of one drop into the pocket formed by gently pulling down the lower eyelid, not touching the bottle to the eye, and eye closure and nasolacrimal occlusion after administration to reduce systemic absorption [1, 2]. If eye drops are instilled improperly it may decrease the therapeutic response, enhance systemic side effects and cause harm to the eye (i.e. bottle contamination or ocular trauma by touching the eye with the bottle tip).

While seemingly a simple task, the correct administration of eye drops may pose problems for patients. In contrast with the numerous reports evaluating administration technique of other non-oral dosage forms such as inhaler devices [3], the amount of literature on eye drop administration is quite limited [4-13]. Previous studies have shown that patients perform relatively poor when instilling eye drops [4-13]. These studies were done in glaucoma patients recruited from eye 
clinics or private ophthalmology practices, mostly in the United States. However, data from a broad patient population in primary care are currently lacking. Therefore, we have recruited a large sample of eye drop users (irrespective of their eye condition) from community pharmacies, and evaluated their eye drop performance. In addition, we assessed patientreported problems with eye drop instillation and previous education on drop technique.

\section{Methods}

\section{Study design}

This cross-sectional, observational study was carried out between December 2014 and May 2015 in 136 community pharmacies in Belgium. Approval for the study was granted by the Ethics Committee of Ghent University Hospital, and all patients gave written informed consent.

\section{Participants}

Patients purchasing prescription or nonprescription medicinal eye drops (Anatomical Therapeutic Chemical classification: S01, except artificial tears (which is S01XA20)) were approached consecutively and invited to participate in the study. They were eligible when meeting the following inclusion criteria: being age 18 years or older, using the eye drops for at least one month (to ensure that patients were already familiar with eye drop instillation), and having sufficient knowledge of Dutch language. Patients who did not normally self-instil their eye drops could also be included; in that case the individual responsible for eye drop administration was invited to demonstrate the technique used. It was planned to recruit five patients from each of the pharmacies.

\section{Data collection}

Data collection took place in the pharmacy or at the patient's home, according to patient preference. Participants completed a self-administered questionnaire developed by the multidisciplinary research team (pharmacists, an ophthalmologist, a general practitioner and a clinical pharmacologist) on the basis of literature and the team's knowledge about the topic. The questionnaire was piloted, before use, in one community pharmacy. It collected the following information: demographics, visual acuity (assessed by a Visual Analogue Scale (VAS) ranging from 0, worst possible eyesight, to 10 , best possible eyesight), current eye disease, current eye medication (brand name) and whether they shake the bottle before use, awareness of limited shelflife of eye drops after opening, perceived difficulty with eye drop administration (assessed by a VAS ranging from 0 , very easy, to 10 , extremely difficult), problems with eye drop instillation and previous education on eye drop instillation technique.

Participants were then asked to demonstrate their eye drop technique. Before the actual demonstration, they were orally questioned about some practical issues related to eye drop administration: hand washing prior to administration, removing contact lenses before using eye drops, and waiting at least $5 \mathrm{~min}$ between instilling multiple eye drops at the same time. Next, patients were asked to show how they normally instil eye drops using a bottle of artificial tears (Optive Fusion ${ }^{\circledR}$, Allergan). Patients who used drops in only one eye were asked to demonstrate drop instillation into this eye. Patients who used drops in both eyes demonstrated the technique in one eye of choice. The technique was directly observed by the pharmacist and scored using a checklist based on a patient education brochure of the American Academy of Ophthalmology [1]. The items of the checklist can be seen in Table 1 (section 'Direct observation of instillation technique'). Before the start of the study, pharmacists received a training session on how to use the

Table 1 Evaluation of eye drop instillation technique

\begin{tabular}{lr}
\hline & $N(\%)$ \\
\hline Questions before the demonstration & \\
Do you wash hands prior to using eye drops? & $231(34.1)$ \\
$\quad$ Always & $204(30.1)$ \\
$\quad$ Sometimes & $243(35.8)$ \\
$\quad$ Never & $17 / 18^{\mathrm{a}}(94.4)$ \\
Do you remove contact lenses before using & \\
eye drops? & \\
Do you wait 5 min between instilling multiple eye & $143 / 189^{\mathrm{b}}(75.7)$ \\
drops at the same time & \\
Direct observation of instillation technique & $670(98.8)$ \\
Removes the cap of the bottle & $622(91.7)$ \\
Does not touch the dropper tip & $619(91.3)$ \\
Tilts the head back slightly & $564(83.2)$ \\
Pulls lower eyelid away from the eye to form & \\
a pocket & $552(81.9)$ \\
Holds the dropper tip directly over the & \\
eyelid pocket & $635(98.0)^{\mathrm{c}}$ \\
At least 1 drop falls into the pocket & $402(59.3)$ \\
Does not touch the bottle to the eye or eyelid & $218(32.2)$ \\
Closes the eye & $36(5.3)^{\mathrm{d}}$ \\
Performs nasolacrimal occlusion for at least 1 min & $20(2.9)$ \\
Patients performing correctly all steps & \\
\hline
\end{tabular}

${ }^{a}$ No. of contact lens wearers

${ }^{\mathrm{b}}$ No. of patients using multiple eye drops at the same time

${ }^{\mathrm{c}} 95(14.0 \%)$ patients required more than 1 attempt to instil at least 1 drop in the pocket

${ }^{\mathrm{d}}$ An additional $40(5.9 \%)$ patients also performed nasolacrimal occlusion, but for less than $1 \mathrm{~min}$ 
Fig. 1 Flow scheme of the participant recruitment process
Assessed for eligibility ( $n=1804)$

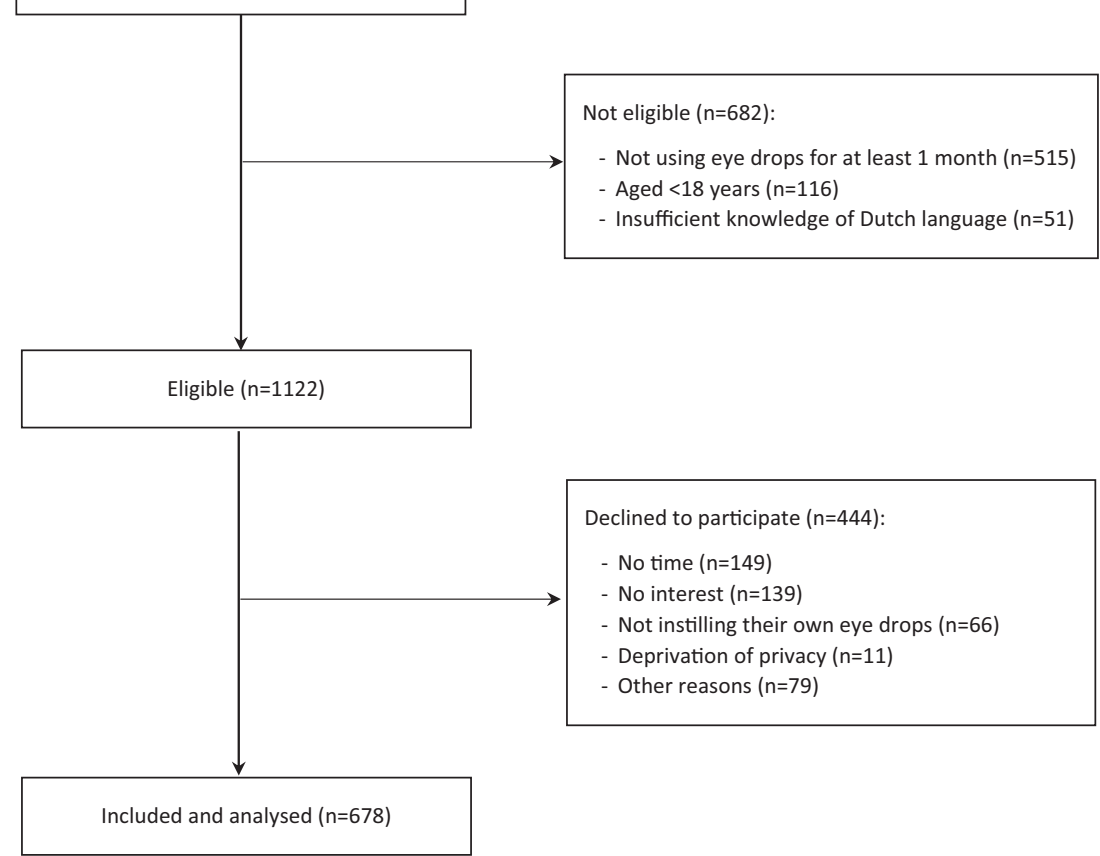

checklist, including videotaped eye drop demonstrations of real patients. After this session, each pharmacist was asked to individually rate three videotaped performances (made available through an online platform) using the checklist and submit their scoring to the research centre. They were subsequently given feedback on their scoring. In addition, we used their scorings to assess inter-rater agreement of the checklist. These results demonstrated that the pharmacists were able to produce consistent scorings when using the checklist (see Supplementary Appendix).

\section{Data analysis}

Descriptive statistics are provided as counts with percentages, means with standard deviations or medians with interquartile ranges as appropriate.

\section{Results}

In the 136 participating pharmacies, 1804 patients were prescreened, of whom, $1122(62.2 \%)$ matched the inclusion criteria. About $60 \%$ of patients $(n=678)$ agreed to participate (Fig. 1). The sample characteristics are detailed in Table 2. Participants had a mean age of 68.9 (SD 12.4) years. Antiglaucoma preparations (used by $88.2 \%$ of patients) and anti-inflammatory agents $(9.6 \%)$ were the most frequently used eye medications.

\section{Drop technique demonstration}

Most patients self-instilled their eye drops, while $15 \%$ $(n=99)$ had their drops administered by another person. About one third of participants $(n=243 ; 35.8 \%)$ reported to never wash hands prior to using eye drops (Table 1). Twenty-four percent of those using multiple eye drops at the same time (46/189) did not wait 5 min between drops. During the demonstration, almost everyone $(n=635$; $98.0 \%$ ) successfully instilled at least one drop in the eye, although $14 \%(n=95)$ required multiple attempts to achieve this. Only $3 \%$ of the sample $(n=20)$ exhibited perfect drop technique, meaning that they performed correctly all the steps. Most common deviations were: touching the bottle to the eye or eyelid $(n=276 ; 40.7 \%)$, and failing to close the eye $(n=460 ; 67.8 \%)$ and perform nasolacrimal occlusion for at least $1 \min (n=642 ; 94.7 \%)$ after drop instillation.

When comparing eye drop performance between patients who self-instil their drops with patients who have their drops administered by another person, we observed statistically significant differences ( $p<0.05$, chi square test) in three steps: 'tilts the head back slightly' $(90.2 \%$ of the selfinstillation group performed this step correctly vs. $98.0 \%$ of the instillation by others group), 'holds the dropper tip directly over the eyelid pocket' $(80.6 \%$ vs. $89.8 \%)$, and 'does not touch the bottle to the eye or eyelid' (54.2\% vs. $88.9 \%$ ). 
Table 2 Sample characteristics

\begin{tabular}{|c|c|}
\hline & $N=678$ \\
\hline Female sex & $398(58.7)$ \\
\hline Age (years), mean $\pm \mathrm{SD}$ & $68.9 \pm 12.4$ \\
\hline \multicolumn{2}{|l|}{ Living situation } \\
\hline Alone & $205(30.2)$ \\
\hline With spouse or partner & $451(66.5)$ \\
\hline With relatives & $20(2.9)$ \\
\hline Other & $2(0.3)$ \\
\hline \multicolumn{2}{|l|}{ Education level } \\
\hline Primary education & $132(19.5)$ \\
\hline Secondary education & $361(53.2)$ \\
\hline Higher education & $185(27.3)$ \\
\hline \multicolumn{2}{|l|}{ Self-rated health ${ }^{a}$} \\
\hline Excellent & $49(7.2)$ \\
\hline Very good & $164(24.2)$ \\
\hline Good & $367(54.1)$ \\
\hline Fair & $90(13.3)$ \\
\hline Poor & $7(1.0)$ \\
\hline \multicolumn{2}{|l|}{ Visual acuity (self-reported on a 0-10 VAS) } \\
\hline Uncorrected, mean \pm SD & $5.5 \pm 2.2$ \\
\hline Corrected, mean $\pm \mathrm{SD}$ & $8.2 \pm 1.8$ \\
\hline \multicolumn{2}{|l|}{ Eye disease } \\
\hline Glaucoma & $542\left(79.9^{b}\right)$ \\
\hline Cataract & $114(16.8)$ \\
\hline Eye infection & $37(5.5)$ \\
\hline Eye allergy & $33(4.9)$ \\
\hline Other & $87(12.8)$ \\
\hline \multicolumn{2}{|l|}{ Eye medication } \\
\hline Antiglaucoma preparations & $598(88.2)$ \\
\hline Beta-blockers & $409(60.3)$ \\
\hline Prostaglandin analogues & $272(40.1)$ \\
\hline Carbonic anhydrase inhibitors & $74(10.9)$ \\
\hline Sympathomimetics & $37(5.5)$ \\
\hline Parasympathomimetics & $17(2.5)$ \\
\hline Anti-inflammatory agents & $65(9.6)$ \\
\hline Corticosteroids & $50(7.4)$ \\
\hline NSAID & $20(2.9)$ \\
\hline Decongestants and antiallergics & $28(4.1)$ \\
\hline Combination of antiinfectives and anti-inflammatory agents & $23(3.4)$ \\
\hline Antiinfectives & $13(1.9)$ \\
\hline Mydriatics and cycloplegics & $4(0.6)$ \\
\hline
\end{tabular}

Data are presented as $N(\%)$ or mean $\pm \mathrm{SD}$

VAS Visual Analogue Scale

${ }^{\mathrm{a}} 1$ missing value

${ }^{\mathrm{b}}$ Some patients reported $>1$ diagnosis

\section{Questionnaire}

Patients generally perceived drop instillation as not difficult and assigned a median difficulty score of 1 on a $0-10$ VAS (IQR 3) (Table 3). Seventy percent of patients $(n=474)$ graded difficulty as $0-2$ out of $10,13.7 \%(n=93)$ as $3-5$ out of 10 , and $16.4 \%(n=111)$ as more than 5 out of 10 . Forty percent reported at least one problem with eye drop instillation. Most common problems were: difficulties with
Table 3 Patient-reported problems with eye drop instillation and previous education on eye drop instillation technique

Patient-reported problems with eye drop instillation

Perceived difficulty of eye drop instillation (on a $0-10$

$1.0(3.0)$

VAS), median (IQR)

Self-reported problems with eye drop instillation ${ }^{\mathrm{a}}$

Patients with at least one problem 269 (39.7)

Difficult to get drop in eye 124 (18.3)

Too many drops come out $\quad 99$ (14.6)

Hard to squeeze the bottle 83 (12.2)

Not sure drop actually gets in eye 44 (6.5)

Hard to open bottle 33 (4.9)

Too much blinking $30(4.4)$

Hard to hold bottle over eye 27 (4.0)

Hard to tilt back the head 17 (2.5)

Shaky hands $15(2.2)$

Other 27 (4.0)

Ever reported these problems to a health professional $\quad 61 / 269$ (22.7)

Type of health professional ${ }^{\mathrm{a}}$

$\begin{array}{lc}\text { Ophthalmologist } & 42 / 61(68.9) \\ \text { Community pharmacist } & 11 / 61(18.0) \\ \text { Nurse } & 6 / 61(9.8) \\ \text { General practitioner } & 4 / 61(6.6) \\ \text { Other } & 5 / 61(8.2)\end{array}$

Action taken by health professional to resolve the problem

$\begin{array}{lc}\text { Extra education on eye drop technique } & 18 / 61(29.5) \\ \text { None } & 11 / 61(18.0) \\ \text { Other }{ }^{\mathrm{b}} & 19 / 61(31.1) \\ \text { Missing } & 13 / 61(21.3)\end{array}$

Eye drop dispensing aids

Knows it exists $62(9.1)$

Uses it $\quad 5(0.7)$

Education on eye drop instillation technique

Ever received eye drop technique education 346 (51.0)

Eye drop technique education was provided by ${ }^{\mathrm{a}}$

Ophthalmologist

$282 / 346(82.0)$

Community pharmacist

$47 / 346(13.7)$

Nurse

General practitioner

$26 / 346(7.6)$

Other

$6 / 346(1.7)$

$22 / 346(6.4)$

Eye drop technique education included

Verbal instruction only

$175 / 346(50.6)$

Verbal instruction and physical demonstration

$145 / 346(41.9)$

Did not remember

$26 / 346(7.5)$

Data are presented as $N(\%)$, unless indicated otherwise

$I Q R$ interquartile range

${ }^{\text {aT }}$ Total percentage exceeds $100 \%$ because some patients selected multiple responses

${ }^{b}$ Various actions, e.g. arranging a home care nurse to administer the eye drops, prescribing another brand with an easier to use bottle, informing the patient that putting too much drops in the eye is not harmful, advising the use of an eye drop aid, ...

getting a drop in the eye $(n=124 ; 18.3 \%)$, too many drops coming out of the bottle $(n=99 ; 14.6 \%)$, and difficulty squeezing the bottle $(n=83 ; 12.2 \%)$. Only a minority (61/ $269 ; 22.7 \%$ ) ever reported these problems to a health professional, mostly to an ophthalmologist $(42 / 61 ; 68.9 \%)$. Eye 
drop dispensing aids appeared to be poorly known $(n=62$; $9.1 \%)$ and even less actually used $(n=5 ; 0.7 \%)$. About half of the sample $(n=346)$ recalled having had education in eye drop instillation technique; most of these (282/346; $82.0 \%$ ) received tuition from an ophthalmologist.

Our survey also revealed that $20 \%$ of ophthalmic suspensions (36/182) were not shaken before use, and that most patients $(n=580 ; 85.5 \%)$ knew that eye drops have a limited shelf-life after opening.

\section{Discussion}

\section{Main findings}

Our study showed that the vast majority of patients was able to successfully instil at least one drop into the eye. This is a reassuring finding, as getting the eye drops in the eye is key for achieving therapeutic response. However, optimal pharmacotherapy also requires that unwanted effects are kept to a minimum. On this issue, we found substantial room for improvement: $40 \%$ of patients touched the bottle tip to the eye and only a minority performed eye closure and nasolacrimal occlusion after instilling the drops.

It is generally recommended to avoid contact between the bottle tip and the eye as this can lead to eye drop contamination. Several reports have demonstrated that ophthalmic solutions can actually become contaminated with microorganisms during repeated use [14-18]. For example, a recent study detected microbial contamination in $24 \%$ of multiuse eye drops applied by glaucoma patients at home [18]. Although eye drop contamination seems not to cause frequent eye infections, there is a small but present risk (conjunctivitis, keratitis and endophthalmitis have been reported [16, 17, 19-22]). In this context, it is noteworthy that touching the ocular surface with the bottle tip might also cause mechanical damage to the cornea. This risk is probably small, but it is another reason to avoid bottle-eye contact. Eye drop dispensing aids could be a useful tool to help patients instil eye drops without touching the eye. The $\mathrm{Xal}_{\text {-ease }}{ }^{\circledR}$ is the best studied device and showed to significantly reduce the problem of bottle-eye contact $[23,24]$. Nevertheless, our findings indicated that patient awareness of eye drop dispensing aids is poor, so implementing these devices will require additional efforts from healthcare providers.

Eye closure and nasolacrimal occlusion are simple techniques that not only increase the ocular bioavailability of ophthalmic preparations but also reduce the probability of systemic side effects $[25,26]$. The amount of systemic absorption of eye drops can be significant [27]. This is particularly relevant in case of beta blocker eye drops (of note, $60 \%$ of our sample used these). Major systemic side effects, such as bradycardia, worsening of heart failure and bronchospasm, have been reported [28]. In addition, the systemically absorbed fraction of ophthalmic betablockers can interact with other drugs, e.g. inhaled beta- 2 agonists (reducing their bronchodilatory effect) and verapamil (enhancing the hypotensive effect) [29]. The clinical relevance of nasolacrimal occlusion was demonstrated for several ocular drugs [26, 30-32]. For instance, one study found that nasolacrimal occlusion after instillation of timolol $0.5 \%$ drops reduced systemic drug absorption by about $60 \%$ [26]. In our sample, only one third of patients closed their eye after drop instillation and as little as $5 \%$ pressed on the nasolacrimal punctum for at least $1 \mathrm{~min}$.

Another clinically relevant finding is that one-fifth of ophthalmic suspensions were not shaken prior to use. At first glance this might seem rather trivial but shaking suspensions before use is crucial to ensure dose uniformity. Because suspensions contain drug particles that are undissolved, shaking is necessary to distribute the drug throughout the vehicle. If suspensions are not shaken, the first few doses will be underdosed (which may lead to therapeutic failure) while the last doses will be overdosed (potentially causing drug toxicity). This was demonstrated by an experimental study of prednisolone acetate ophthalmic suspensions, which revealed drug doses ranging from 21 to $181 \%$ of the labelled content [33].

Importantly, we found that only half of patients had received education by any healthcare professional regarding the proper instillation technique of eye drops. This may be an underestimation due to recall bias. Even so, there is much room for improvement as every patient deserves to receive tuition on how to use his medication. Only a small number of patients had received instructions from their pharmacist, indicating a need for improved pharmacy services. Patients should systematically be taught how to instil their eye drops when they are first dispensed eye drops. At subsequent refills, pharmacists should proactively ask about problems with administering eye drops as the current study showed that only a minority of problems were actually reported to a health professional.

\section{Comparison with other studies}

Our findings are generally consistent with previous studies showing suboptimal eye drop technique in selected populations. These studies mainly focused on the key criteria of successful instillation, i.e. getting one drop in the eye without touching the bottle tip to the eye. Our observed prevalence of missing the eye $(2 \%)$ is situated in the lower end of the prevalence rates seen in other studies (ranging from 0 to 25\%) [4, 7-9, 11, 12], whereas our prevalence of bottle-eye contact $(40 \%)$ falls toward the 
upper end of the prevalence reported in previous studies (ranging from 15 to $80 \%$ ) $[4,7-10,12]$.

\section{Strengths and limitations of the study}

To our knowledge this study is the first to investigate eye drop technique and patient-reported problems with eye drop instillation in a primary care setting. We attempted to recruit a patient sample as representative as possible, using a high number of community-based recruitment centres (pharmacies) and broad patient inclusion criteria (i.e. including any eye drop user irrespective of eye condition, and not excluding patients relying on another person to instil their eye drops). Whereas previous studies only assessed key steps required for eye drop instillation, the present study documented all steps recommended by the American Academy of Ophthalmology. This added, for example, information on the implementation of eye closure and nasolacrimal occlusion.

This study also has some limitations. First, eye drop technique was assessed by 136 different evaluators. Ideally, eye drop performances would have been videotaped and subsequently scored by one or two evaluators. However, videotaping was considered to be not practically feasible due to the high number of study centres ( $n$ $=136$ ). By using a checklist and thorough training on how to apply the checklist (including videos of real patients), inter-rater variability was reduced as much as possible. This was objectively confirmed by calculation of inter-rater agreement, which showed consistency among raters. However, the fact that we used only three videos to determine inter-rater agreement may be regarded as a limitation. Second, there are some factors that may have impacted patients' eye drop technique. When people know they are being observed they may act differently than normal. This may have had a positive or negative impact on eye drop performance. Patients did not demonstrate eye drop technique with their own drops but with a bottle of artificial tears provided by the researchers. This may have affected eye drop performance. Participants were asked to demonstrate their instillation technique in one eye of their choice. This may also have biased results as patients may have chosen their best eye. Third, the questionnaire collected self-reported data, which may be subject to recall bias (e.g. regarding previous education on eye drop technique). Fourth, a relatively high number of potential participants refused to participate in the study (40\%). Potential bias caused by those who refused participation could not be assessed as our Ethics Committee prohibits data collection in study refusers. However, we did record reasons for refusal ('no time' and 'no interest' were the most commonly given reasons).

\section{Summary}

\section{What was known before}

- Correct administration of eye drops may pose problems for patients

- Incorrect eye drop instillation may decrease therapeutic response, enhance systemic side effects and cause harm to the eye

\section{What this study adds}

- Most patients were able to successfully instil at least one drop into the eye

- Most common errors were: touching the bottle to the eye or eyelid, and failing to close the eye and perform nasolacrimal occlusion

- Many patients had never received education regarding correction administration of eye drops

Acknowledgements The authors would like to thank the participating patients and pharmacists. They also thank Allergan Belgium for donating the artificial tears.

\section{Compliance with ethical standards}

Conflict of interest The authors declare that they have no conflict of interest.

Publisher's note Springer Nature remains neutral with regard to jurisdictional claims in published maps and institutional affiliations.

\section{References}

1. Patient education brochure 'Eye drops-a closer look'. San Francisco: American Academy of Ophthalmology; 2011.

2. Joint Formulary Committee (2018). British National Formulary (online version). London: BMJ Publishing Group Ltd and Royal Pharmaceutical Society. Accessed 6 Jan 2019.

3. Sanchis J, Gich I, Pedersen S. Systematic review of errors in inhaler use: has patient technique improved over time? Chest. 2016;150:394-406.

4. Brown MM, Brown GC, Spaeth GL. Improper topical selfadministration of ocular medication among patients with glaucoma. Can J Ophthalmol J Canadien D'ophtalmologie. 1984;19: $2-5$.

5. Hennessy AL, Katz J, Covert D, Kelly CA, Suan EP, Speicher MA, et al. A video study of drop instillation in both glaucoma and retina patients with visual impairment. Am J Ophthalmol. 2011; 152:982-8.

6. Hennessy AL, Katz J, Covert D, Protzko C, Robin AL. Videotaped evaluation of eyedrop instillation in glaucoma patients with visual impairment or moderate to severe visual field loss. Ophthalmology. 2010;117:2345-52.

7. Kass MA, Hodapp E, Gordon M, Kolker AE, Goldberg I. Patient administration of eyedrops: observation. Part II. Ann Ophthalmol. 1982;14:889-93. 
8. Kholdebarin R, Campbell RJ, Jin YP, Buys YM. Multicenter study of compliance and drop administration in glaucoma. Can $\mathrm{J}$ Ophthalmol J Canadien D'ophtalmologie. 2008;43:454-61.

9. Schwartz GF, Hollander DA, Williams JM. Evaluation of eye drop administration technique in patients with glaucoma or ocular hypertension. Curr Med Res Opin. 2013;29:1515-22.

10. Sleath B, Blalock S, Covert D, Stone JL, Skinner AC, Muir K, et al. The relationship between glaucoma medication adherence, eye drop technique, and visual field defect severity. Ophthalmology. 2011;118:2398-402.

11. Stone JL, Robin AL, Novack GD, Covert DW, Cagle GD. An objective evaluation of eyedrop instillation in patients with glaucoma. Arch Ophthalmol. 2009;127:732-6.

12. Tatham AJ, Sarodia U, Gatrad F, Awan A. Eye drop instillation technique in patients with glaucoma. Eye. 2013;27:1293-8.

13. Gao X, Yang Q, Huang W, Chen T, Zuo C, Li X, et al. Evaluating eye drop instillation technique and its determinants in glaucoma patients. J Ophthalmol. 2018;2018:1376020.

14. Geyer O, Bottone EJ, Podos SM, Schumer RA, Asbell PA. Microbial contamination of medications used to treat glaucoma. Br J Ophthalmol. 1995;79:376-9.

15. Jokl DH, Wormser GP, Nichols NS, Montecalvo MA, Karmen CL. Bacterial contamination of ophthalmic solutions used in an extended care facility. Br J Ophthalmol. 2007;91:1308-10.

16. Porges Y, Rothkoff L, Glick J, Cohen S. Sterility of glaucoma medications among chronic users in the community. J Ocul Pharmacol Therapeutics. 2004;20:123-8.

17. Schein OD, Hibberd PL, Starck T, Baker AS, Kenyon KR. Microbial contamination of in-use ocular medications. Arch Ophthalmol. 1992;110:82-5.

18. Teuchner B, Wagner J, Bechrakis NE, Orth-Holler D, Nagl M. Microbial contamination of glaucoma eyedrops used by patients compared with ocular medications used in the hospital. Medicine. 2015;94:e583.

19. Lalitha P, Das M, Purva PS, Karpagam R, Geetha M, Lakshmi Priya J, et al. Postoperative endophthalmitis due to Burkholderia cepacia complex from contaminated anaesthetic eye drops. Br J Ophthalmol. 2014;98:1498-502.

20. Penland RL, Wilhelmus KR. Stenotrophomonas maltophilia ocular infections. Arch Ophthalmol. 1996;114:433-6.
21. Schein OD, Wasson PJ, Boruchoff SA, Kenyon KR. Microbial keratitis associated with contaminated ocular medications. Am J Ophthalmol. 1988;105:361-5.

22. Templeton WC 3rd, Eiferman RA, Snyder JW, Melo JC, Raff MJ. Serratia keratitis transmitted by contaminated eyedroppers. Am J Ophthalmol. 1982;93:723-6.

23. Gomes BF, Lordello M, Celli LF, Santhiago MR, Moraes HV. Comparison of eyedrop instillation technique with and without a delivery device in inexperienced patients. Eur J Ophthalmol. 2016;26:594-7.

24. Nordmann JP, Baudouin C, Bron A, Denis P, Rouland JF, Sellem E, et al. Xal-Ease: impact of an ocular hypotensive delivery device on ease of eyedrop administration, patient compliance, and satisfaction. Eur J Ophthalmol. 2009;19:949-56.

25. Urtti A, Salminen L. Minimizing systemic absorption of topically administered ophthalmic drugs. Surv Ophthalmol. 1993; $37: 435-56$.

26. Zimmerman TJ, Kooner KS, Kandarakis AS, Ziegler LP. Improving the therapeutic index of topically applied ocular drugs. Arch Ophthalmol. 1984;102:551-3.

27. Järvinen $K$, Järvinen $T$, Urtti A. Ocular absorption following topical delivery. Adv Drug Deliv Rev. 1995;16:3-19.

28. Lama PJ. Systemic adverse effects of beta-adrenergic blockers: an evidence-based assessment. Am J Ophthalmol. 2002;134:749-60.

29. Preston CL. Stockley's drug interactions. 11th ed. Pharmaceutical Press; 2016.

30. Ellis PP, Wu PY, Pfoff DS, Bloedow DC, Riegel MR. Effect of nasolacrimal occlusion on timolol concentrations in the aqueous humor of the human eye. J Pharm Sci. 1992;81: 219-20.

31. Zimmerman TJ, Sharir M, Nardin GF, Fuqua M. Therapeutic index of pilocarpine, carbachol, and timolol with nasolacrimal occlusion. Am J Ophthalmol. 1992;114:1-7.

32. Zimmerman TJ, Sharir M, Nardin GF, Fuqua M. Therapeutic index of epinephrine and dipivefrin with nasolacrimal occlusion. Am J Ophthalmol. 1992;114:8-13.

33. Stringer W, Bryant R. Dose uniformity of topical corticosteroid preparations: difluprednate ophthalmic emulsion $0.05 \%$ versus branded and generic prednisolone acetate ophthalmic suspension 1\%. Clin Ophthalmol. 2010;4:1119-24. 